\title{
A new Hardy-Hilbert-type inequality with multiparameters and a best possible constant factor
}

Yanping Shi ${ }^{1 *}$ and Bicheng Yang ${ }^{2}$

\section{"Correspondence:}

jsdxsysyp1978@163.com

${ }^{1}$ Normal College of Jishou

University, Jishou, Hunan 416000,

P.R. China

Full list of author information is

available at the end of the article

\begin{abstract}
By means of weight coefficients and techniques of real analysis, a new Hardy-Hilbert-type inequality with multiparameters and the best possible constant factor is given. The equivalent forms, the operator expression with the norm, and the reverse and some particular inequalities with the best possible constant factors are also considered.
\end{abstract}

MSC: 26D15; 47A07

Keywords: Hardy-Hilbert-type inequality; weight coefficient; equivalent form; reverse; operator

\section{Introduction}

If $p>1, \frac{1}{p}+\frac{1}{q}=1, f(x), g(y) \geq 0, f \in L^{p}\left(\mathbf{R}_{+}\right), g \in L^{q}\left(\mathbf{R}_{+}\right),\|f\|_{p}=\left(\int_{0}^{\infty} f^{p}(x) d x\right)^{\frac{1}{p}}>0,\|g\|_{q}>0$, then we have the following Hardy-Hilbert integral inequality [1]:

$$
\int_{0}^{\infty} \int_{0}^{\infty} \frac{f(x) g(y)}{x+y} d x d y<\frac{\pi}{\sin (\pi / p)}\|f\|_{p}\|g\|_{q}
$$

where, the constant factor $\frac{\pi}{\sin (\pi / p)}$ is the best possible. Assuming that $a_{m}, b_{n} \geq 0, a=$ $\left\{a_{m}\right\}_{m=1}^{\infty} \in l^{p}, b=\left\{b_{n}\right\}_{n=1}^{\infty} \in l^{q},\|a\|_{p}=\left(\sum_{m=1}^{\infty} a_{m}^{p}\right)^{\frac{1}{p}}>0,\|b\|_{q}>0$, we have the following Hardy-Hilbert inequality with the same best possible constant factor $\frac{\pi}{\sin (\pi / p)}[1]$ :

$$
\sum_{m=1}^{\infty} \sum_{n=1}^{\infty} \frac{a_{m} b_{n}}{m+n}<\frac{\pi}{\sin (\pi / p)}\|a\|_{p}\|b\|_{q}
$$

Inequalities (1) and (2) are important in analysis and its applications [1-5].

$$
\text { If } \begin{aligned}
\mu_{i}, v_{j} & >0(i, j \in \mathbf{N}=\{1,2, \ldots\}), \\
U_{m} & :=\sum_{i=1}^{m} \mu_{i}, \quad V_{n}:=\sum_{j=1}^{n} v_{j} \quad(m, n \in \mathbf{N}),
\end{aligned}
$$

\section{Springer}


then we have the following inequality (see Theorem 321 of [1]):

$$
\sum_{m=1}^{\infty} \sum_{n=1}^{\infty} \frac{\mu_{m}^{1 / q} v_{n}^{1 / p} a_{m} b_{n}}{U_{m}+V_{n}}<\frac{\pi}{\sin (\pi / p)}\|a\|_{p}\|b\|_{q}
$$

Replacing $\mu_{m}^{1 / q} a_{m}$ and $v_{n}^{1 / p} b_{n}$ by $a_{m}$ and $b_{n}$ in (4), respectively, we have the following equivalent form of (4):

$$
\sum_{m=1}^{\infty} \sum_{n=1}^{\infty} \frac{a_{m} b_{n}}{U_{m}+V_{n}}<\frac{\pi}{\sin \left(\frac{\pi}{p}\right)}\left(\sum_{m=1}^{\infty} \frac{a_{m}^{p}}{\mu_{m}^{p-1}}\right)^{\frac{1}{p}}\left(\sum_{n=1}^{\infty} \frac{b_{n}^{q}}{v_{n}^{q-1}}\right)^{\frac{1}{q}} .
$$

For $\mu_{i}=v_{j}=1(i, j \in \mathbf{N})$, both (4) and (5) reduce to (2). We call (4) and (5) Hardy-Hilberttype inequalities.

Note The authors of [1] did not prove that (4) is valid with the best possible constant factor.

In 1998, by introducing an independent parameter $\lambda \in(0,1]$ Yang [6] gave an extension of (1) with the kernel $\frac{1}{(x+y)^{\lambda}}$ for $p=q=2$. Following the results of [6], Yang [5] gave some best extensions of (1) and (2) as follows.

If $\lambda_{1}, \lambda_{2} \in \mathbf{R}, \lambda_{1}+\lambda_{2}=\lambda, k_{\lambda}(x, y)$ is a nonnegative homogeneous function of degree $-\lambda$ with $k\left(\lambda_{1}\right)=\int_{0}^{\infty} k_{\lambda}(t, 1) t^{\lambda_{1}-1} d t \in \mathbf{R}_{+}, \phi(x)=x^{p\left(1-\lambda_{1}\right)-1}, \psi(x)=x^{q\left(1-\lambda_{2}\right)-1}, f(x), g(y) \geq 0$,

$$
f \in L_{p, \phi}\left(\mathbf{R}_{+}\right)=\left\{f ;\|f\|_{p, \phi}:=\left(\int_{0}^{\infty} \phi(x)|f(x)|^{p} d x\right)^{\frac{1}{p}}<\infty\right\}
$$

$g \in L_{q, \psi}\left(\mathbf{R}_{+}\right),\|f\|_{p, \phi},\|g\|_{q, \psi}>0$, then

$$
\int_{0}^{\infty} \int_{0}^{\infty} k_{\lambda}(x, y) f(x) g(y) d x d y<k\left(\lambda_{1}\right)\|f\|_{p, \phi}\|g\|_{q, \psi},
$$

where the constant factor $k\left(\lambda_{1}\right)$ is the best possible. Moreover, if $k_{\lambda}(x, y)$ is finite and $k_{\lambda}(x, y) x^{\lambda_{1}-1}\left(k_{\lambda}(x, y) y^{\lambda_{2}-1}\right)$ is decreasing with respect to $x>0(y>0)$, then for $a_{m}, b_{n} \geq 0$,

$$
a \in l_{p, \phi}=\left\{a ;\|a\|_{p, \phi}:=\left(\sum_{n=1}^{\infty} \phi(n)\left|a_{n}\right|^{p}\right)^{\frac{1}{p}}<\infty\right\},
$$

$b=\left\{b_{n}\right\}_{n=1}^{\infty} \in l_{q, \psi},\|a\|_{p, \phi},\|b\|_{q, \psi}>0$, it follows that

$$
\sum_{m=1}^{\infty} \sum_{n=1}^{\infty} k_{\lambda}(m, n) a_{m} b_{n}<k\left(\lambda_{1}\right)\|a\|_{p, \phi}\|b\|_{q, \psi}
$$

where the constant factor $k\left(\lambda_{1}\right)$ is still the best possible.

Clearly, for $\lambda=1, k_{1}(x, y)=\frac{1}{x+y}, \lambda_{1}=\frac{1}{q}, \lambda_{2}=\frac{1}{p}$, inequality (6) reduces to (1), whereas (7) reduces to (2). For $0<\lambda_{1}, \lambda_{2} \leq 1, \lambda_{1}+\lambda_{2}=\lambda$, we set

$$
k_{\lambda}(x, y)=\frac{1}{(x+y)^{\lambda}} \quad\left((x, y) \in \mathbf{R}_{+}^{2}\right) .
$$


Then by (7) it follows that

$$
\sum_{m=1}^{\infty} \sum_{n=1}^{\infty} \frac{a_{m} b_{n}}{(m+n)^{\lambda}}<B\left(\lambda_{1}, \lambda_{2}\right)\|a\|_{p, \phi}\|b\|_{q, \psi}
$$

where the constant $B\left(\lambda_{1}, \lambda_{2}\right)$ is the best possible. Some other results including multidimensional Hilbert-type inequalities are provided in [7-24].

In this paper, by means of weight coefficients and techniques of real analysis, a new Hardy-Hilbert-type inequality with multiparameters and the best possible constant factor is given, which is with the kernel

$$
k_{\lambda}(x, y)=\frac{(\min \{x, y\})^{\alpha}}{(\max \{x, y\})^{\lambda+\alpha}}
$$

similar to (4). The equivalent forms, the operator expression with the norm, the reverse and some particular inequalities with the best possible constant factors are also considered.

\section{An example and some lemmas}

In the following, we make appointment that $\mu_{i}, v_{j}>0(i, j \in \mathbf{N}), U_{m}$ and $V_{n}$ are defined by (3), $p \neq 0,1, \frac{1}{p}+\frac{1}{q}=1, a_{m}, b_{n} \geq 0(m, n \in \mathbf{N})$,

$$
\|a\|_{p, \Phi_{\lambda}}=\left(\sum_{m=1}^{\infty} \Phi_{\lambda}(m) a_{m}^{p}\right)^{\frac{1}{p}}, \quad\|b\|_{q, \Psi_{\lambda}}=\left(\sum_{n=1}^{\infty} \Psi_{\lambda}(n) b_{n}^{q}\right)^{\frac{1}{q}}
$$

where

$$
\Phi_{\lambda}(m):=\frac{U_{m}^{p\left(1-\lambda_{1}\right)-1}}{\mu_{m}^{p-1}}, \quad \Psi_{\lambda}(n):=\frac{V_{n}^{q\left(1-\lambda_{2}\right)-1}}{v_{n}^{q-1}} \quad(m, n \in \mathbf{N}) .
$$

We also set

$$
\widetilde{\Phi}_{\lambda}(m):=\left(1-\theta\left(\lambda_{2}, m\right)\right) \frac{U_{m}^{p\left(1-\lambda_{1}\right)-1}}{\mu_{m}^{p-1}}, \quad \widetilde{\Psi}_{\lambda}(n):=\left(1-\vartheta\left(\lambda_{1}, n\right)\right) \frac{V_{n}^{q\left(1-\lambda_{2}\right)-1}}{v_{n}^{q-1}} \quad(m, n \in \mathbf{N}) .
$$

Note For $0<p<1$ or $p<0$, we still use the formal symbols $\|a\|_{p, \Phi_{\lambda}},\|b\|_{q, \Psi_{\lambda}},\|a\|_{p, \widetilde{\Phi}_{\lambda}}$, and $\|b\|_{q, \widetilde{\Psi}_{\lambda}}$.

Example 1 For $-\alpha<\lambda_{1}, \lambda_{2} \leq 1-\alpha, \lambda_{1}+\lambda_{2}=\lambda$, we set

$$
k_{\lambda}(x, y)=\frac{(\min \{x, y\})^{\alpha}}{(\max \{x, y\})^{\lambda+\alpha}} \quad\left((x, y) \in \mathbf{R}_{+}^{2}\right) .
$$

We find

$$
\begin{aligned}
k\left(\lambda_{1}\right) & =\int_{0}^{\infty} k_{\lambda}(t, 1) t^{\lambda_{1}-1} d t=\int_{0}^{\infty} \frac{(\min \{t, 1\})^{\alpha}}{(\max \{t, 1\})^{\lambda+\alpha}} t^{\lambda_{1}-1} d t \\
& =\int_{0}^{1} t^{\lambda_{1}+\alpha-1} d t+\int_{1}^{\infty} \frac{1}{t^{\lambda+\alpha}} t^{\lambda_{1}-1} d t \\
& =\frac{1}{\lambda_{1}+\alpha}+\frac{1}{\lambda_{2}+\alpha}=\frac{\lambda+2 \alpha}{\left(\lambda_{1}+\alpha\right)\left(\lambda_{2}+\alpha\right)} \in \mathbf{R}_{+} .
\end{aligned}
$$


Since

$$
k_{\lambda}(x, y) \frac{1}{y^{1-\lambda_{2}}}= \begin{cases}\frac{y^{\alpha+\lambda_{2}-1}}{x^{\lambda+\alpha}}, & 0<y<x \\ \frac{x^{\alpha}}{y^{1+\lambda} 1^{+\alpha}}, & y \geq x\end{cases}
$$

for $\lambda_{2} \leq 1-\alpha\left(\lambda_{1}>-\alpha\right), k_{\lambda}(x, y) \frac{1}{y^{1-\lambda_{2}}}$ is decreasing for $y>0$ and strictly decreasing for $y$ large enough. Since

$$
k_{\lambda}(x, y) \frac{1}{x^{1-\lambda_{1}}}= \begin{cases}\frac{x^{\alpha+\lambda_{1}-1}}{y^{\lambda+\alpha}}, & 0<x<y \\ \frac{y^{\alpha}}{x^{1+\lambda_{2}+\alpha}}, & x \geq y\end{cases}
$$

for $\lambda_{1} \leq 1-\alpha\left(\lambda_{2}>-\alpha\right), k_{\lambda}(x, y) \frac{1}{x^{1-\lambda_{1}}}$ is decreasing for $x>0$ and strictly decreasing for $x$ large enough.

In other words, for $-\alpha<\lambda_{1}, \lambda_{2} \leq 1-\alpha, k_{\lambda}(x, y) \frac{1}{y^{1-\lambda_{2}}}\left(k_{\lambda}(x, y) \frac{1}{x^{1-\lambda_{1}}}\right)$ is decreasing for $y>0$ $(x>0)$ and strictly decreasing for $y(x)$ large enough, satisfying $k\left(\lambda_{1}\right) \in \mathbf{R}_{+}$.

Lemma 1 If $g(t)(>0)$ is decreasing in $\mathbf{R}_{+}$, strictly decreasing in $\left[n_{0}, \infty\right)\left(n_{0} \in \mathbf{N}\right)$, and satisfying $\int_{0}^{\infty} g(t) d t \in \mathbf{R}_{+}$, then we have

$$
\int_{1}^{\infty} g(t) d t<\sum_{n=1}^{\infty} g(n)<\int_{0}^{\infty} g(t) d t
$$

Proof Since

$$
\begin{aligned}
& \int_{n}^{n+1} g(t) d t \leq g(n) \leq \int_{n-1}^{n} g(t) d t \quad\left(n=1, \ldots, n_{0}\right), \\
& \int_{n_{0}+1}^{n_{0}+2} g(t) d t<g\left(n_{0}+1\right)<\int_{n_{0}}^{n_{0}+1} g(t) d t
\end{aligned}
$$

it follows that

$$
0<\int_{1}^{n_{0}+2} g(t) d t<\sum_{n=1}^{n_{0}+1} g(n)<\sum_{n=1}^{n_{0}+1} \int_{n-1}^{n} g(t) d t=\int_{0}^{n_{0}+1} g(t) d t<\infty .
$$

In the same way, we have

$$
0<\int_{n_{0}+2}^{\infty} g(t) d t \leq \sum_{n=n_{0}+2}^{\infty} g(n) \leq \int_{n_{0}+1}^{\infty} g(t) d t<\infty
$$

Adding these two inequalities, we have (10).

Lemma 2 Let $-\alpha<\lambda_{1}, \lambda_{2} \leq 1-\alpha, \lambda_{1}+\lambda_{2}=\lambda$, and $k\left(\lambda_{1}\right)$ be as in (9). Define the following weight coefficients:

$$
\omega\left(\lambda_{2}, m\right):=\sum_{n=1}^{\infty} \frac{\left(\min \left\{U_{m}, V_{n}\right\}\right)^{\alpha}}{\left(\max \left\{U_{m}, V_{n}\right\}\right)^{\lambda+\alpha}} \frac{U_{m}^{\lambda_{1}} v_{n}}{V_{n}^{1-\lambda_{2}}}, \quad m \in \mathbf{N}
$$




$$
\varpi\left(\lambda_{1}, n\right):=\sum_{m=1}^{\infty} \frac{\left(\min \left\{U_{m}, V_{n}\right\}\right)^{\alpha}}{\left(\max \left\{U_{m}, V_{n}\right\}\right)^{\lambda+\alpha}} \frac{V_{n}^{\lambda_{2}} \mu_{m}}{U_{m}^{1-\lambda_{1}}}, \quad n \in \mathbf{N}
$$

Then, we have the following inequalities:

$$
\begin{array}{ll}
\omega\left(\lambda_{2}, m\right)<k\left(\lambda_{1}\right) & \left(-\alpha<\lambda_{2} \leq 1-\alpha, \lambda_{1}>-\alpha ; m \in \mathbf{N}\right), \\
\varpi\left(\lambda_{1}, n\right)<k\left(\lambda_{1}\right) & \left(-\alpha<\lambda_{1} \leq 1-\alpha, \lambda_{2}>-\alpha ; n \in \mathbf{N}\right) .
\end{array}
$$

Proof We set $\mu(t):=\mu_{m}, t \in(m-1, m](m \in \mathbf{N}) ; v(t):=v_{n}, t \in(n-1, n](n \in \mathbf{N})$, and

$$
U(x):=\int_{0}^{x} \mu(t) d t \quad(x \geq 0), \quad V(y):=\int_{0}^{y} v(t) d t \quad(y \geq 0) .
$$

Then by (3) it follows that $U(m)=U_{m}, V(n)=V_{n}(m, n \in \mathbf{N})$. For $x \in(m-1, m), U^{\prime}(x)=$ $\mu(x)=\mu_{m}(m \in \mathbf{N})$; for $y \in(n-1, n), V^{\prime}(y)=v(y)=v_{n}(n \in \mathbf{N})$. Since $V(y)$ is strictly increasing in $(n-1, n],-\alpha<\lambda_{2} \leq 1-\alpha, \lambda_{1}>-\alpha$, in view of Example 1 and Lemma 1, we find

$$
\begin{aligned}
\omega\left(\lambda_{2}, m\right) & =\sum_{n=1}^{\infty} \int_{n-1}^{n} \frac{\left(\min \left\{U_{m}, V_{n}\right\}\right)^{\alpha}}{\left(\max \left\{U_{m}, V_{n}\right\}\right)^{\lambda+\alpha}} \frac{U_{m}^{\lambda_{1}}}{V_{n}^{1-\lambda_{2}}} V^{\prime}(y) d y \\
& <\sum_{n=1}^{\infty} \int_{n-1}^{n} \frac{\left(\min \left\{U_{m}, V(y)\right\}\right)^{\alpha}}{\left(\max \left\{U_{m}, V(y)\right\}\right)^{\lambda+\alpha}} \frac{U_{m}^{\lambda_{1}}}{V^{1-\lambda_{2}}(y)} V^{\prime}(y) d y .
\end{aligned}
$$

Setting $t=\frac{V(y)}{U_{m}}$, we obtain $V^{\prime}(y) d y=U_{m} d t$ and

$$
\begin{aligned}
\omega\left(\lambda_{2}, m\right) & <\sum_{n=1}^{\infty} \int_{\frac{V(n-1)}{U_{m}}}^{\frac{V(n)}{U_{m}}} \frac{(\min \{1, t\})^{\alpha}}{(\max \{1, t\})^{\lambda+\alpha}} t^{\lambda_{2}-1} d t \\
& =\int_{0}^{\frac{V(\infty)}{U_{m}}} \frac{(\min \{1, t\})^{\alpha}}{(\max \{1, t\})^{\lambda+\alpha}} t^{\lambda_{2}-1} d t \\
& \leq \int_{0}^{\infty} \frac{(\min \{1, t\})^{\alpha}}{(\max \{1, t\})^{\lambda+\alpha}} t^{\lambda_{2}-1} d t=k\left(\lambda_{1}\right) .
\end{aligned}
$$

Hence, we have (13). In the same way, we have (14).

Lemma 3 Let $-\alpha<\lambda_{1}, \lambda_{2} \leq 1-\alpha, \lambda_{1}+\lambda_{2}=\lambda, \lambda_{1}+\lambda_{2}=\lambda, k\left(\lambda_{1}\right)$ be as in (9), $m_{0}, n_{0} \in \mathbf{N}$, $\mu_{m} \geq \mu_{m+1}\left(m \in\left\{m_{0}, m_{0}+1, \ldots\right\}\right), v_{n} \geq v_{n+1}\left(n \in\left\{n_{0}, n_{0}+1, \ldots\right\}\right), U(\infty)=V(\infty)=\infty$. Then (i) for $m, n \in \mathbf{N}$, we have

$$
\begin{array}{lc}
k\left(\lambda_{1}\right)\left(1-\theta\left(\lambda_{2}, m\right)\right)<\omega\left(\lambda_{2}, m\right) & \left(-\alpha<\lambda_{2} \leq 1-\alpha, \lambda_{1}>-\alpha\right), \\
k\left(\lambda_{1}\right)\left(1-\vartheta\left(\lambda_{1}, n\right)\right)<\varpi\left(\lambda_{1}, n\right) & \left(-\alpha<\lambda_{1} \leq 1-\alpha, \lambda_{2}>-\alpha\right),
\end{array}
$$

where, $\theta\left(\lambda_{2}, m\right)=O\left(\frac{1}{U_{m}^{\lambda_{2}+\alpha}}\right) \in(0,1), \vartheta\left(\lambda_{1}, n\right)=O\left(\frac{1}{V_{n}^{\lambda_{1}+\alpha}}\right) \in(0,1)$;

(ii) for any $a>0$, we have

$$
\sum_{m=1}^{\infty} \frac{\mu_{m}}{U_{m}^{1+a}}=\frac{1}{a}\left(\frac{1}{U_{m_{0}}^{a}}+a O(1)\right)
$$




$$
\sum_{n=1}^{\infty} \frac{v_{n}}{V_{n}^{1+a}}=\frac{1}{a}\left(\frac{1}{V_{n_{0}}^{a}}+a \widetilde{O}(1)\right)
$$

Proof Since $v_{n} \geq v_{n+1}\left(n \geq n_{0}\right),-\alpha<\lambda_{2} \leq 1-\alpha, \lambda_{1}>-\alpha$, and $V(\infty)=\infty$, by Lemma 1 we have

$$
\begin{aligned}
\omega\left(\lambda_{2}, m\right) & \geq \sum_{n=n_{0}}^{\infty} \frac{\left(\min \left\{U_{m}, V_{n}\right\}\right)^{\alpha}}{\left(\max \left\{U_{m}, V_{n}\right\}\right)^{\lambda+\alpha}} \frac{U_{m}^{\lambda_{1}}}{V_{n}^{1-\lambda_{2}}} v_{n+1} \\
& =\sum_{n=n_{0}}^{\infty} \int_{n}^{n+1} \frac{\left(\min \left\{U_{m}, V_{n}\right\}\right)^{\alpha}}{\left(\max \left\{U_{m}, V_{n}\right\}\right)^{\lambda+\alpha}} \frac{U_{m}^{\lambda_{1}}}{V_{n}^{1-\lambda_{2}}} V^{\prime}(y) d y \\
& >\sum_{n=n_{0}}^{\infty} \int_{n}^{n+1} \frac{\left(\min \left\{U_{m}, V(y)\right\}\right)^{\alpha}}{\left(\max \left\{U_{m}, V(y)\right\}\right)^{\lambda+\alpha}} \frac{U_{m}^{\lambda_{1}}}{V^{1-\lambda_{2}}(y)} V^{\prime}(y) d y \\
& =\sum_{n=n_{0}}^{\infty} \int_{\frac{V(n)}{U_{m}}}^{\frac{V(n+1)}{U_{m}}} \frac{(\min \{1, t\})^{\alpha}}{(\max \{1, t\})^{\lambda+\alpha}} t^{\lambda_{2}-1} d t \\
& =\int_{\frac{V\left(n_{0}\right)}{U_{m}}}^{\infty} \frac{(\min \{1, t\})^{\alpha}}{(\max \{1, t\})^{\lambda+\alpha}} t^{\lambda_{2}-1} d t=k\left(\lambda_{1}\right)\left(1-\theta\left(\lambda_{2}, m\right)\right),
\end{aligned}
$$

where

$$
\theta\left(\lambda_{2}, m\right):=\frac{1}{k\left(\lambda_{1}\right)} \int_{0}^{\frac{V\left(n_{0}\right)}{u_{m}}} \frac{(\min \{1, t\})^{\alpha}}{(\max \{1, t\})^{\lambda+\alpha}} t^{\lambda_{2}-1} d t \in(0,1) .
$$

For $U_{m}>V\left(n_{0}\right)$, we obtain

$$
\begin{aligned}
0 & <\theta\left(\lambda_{2}, m\right)=\frac{1}{k\left(\lambda_{1}\right)} \int_{0}^{\frac{V\left(n_{0}\right)}{U_{m}}} t^{\lambda_{2}+\alpha-1} d t \\
& =\frac{1}{\left(\lambda_{2}+\alpha\right) k\left(\lambda_{1}\right)}\left(\frac{V_{n_{0}}}{U_{m}}\right)^{\lambda_{2}+\alpha},
\end{aligned}
$$

and then $\theta\left(\lambda_{2}, m\right)=O\left(\frac{1}{U_{m}^{\lambda_{2}+\alpha}}\right)$. Hence, we have (17).

In the same way, since $\mu_{m} \geq \mu_{m+1}\left(m \geq m_{0}\right),-\alpha<\lambda_{1} \leq 1-\alpha, \lambda_{2}>-\alpha$, and $U(\infty)=\infty$, we have

$$
\begin{aligned}
& \varpi\left(\lambda_{1}, n\right) \geq \sum_{m=m_{0}}^{\infty} \frac{\left(\min \left\{U_{m}, V_{n}\right\}\right)^{\alpha}}{\left(\max \left\{U_{m}, V_{n}\right\}\right)^{\lambda+\alpha}} \frac{V_{n}^{\lambda_{2}} \mu_{m+1}}{U_{m}^{1-\lambda_{1}}} \\
&=\sum_{m=m_{0}}^{\infty} \int_{m}^{m+1} \frac{\left(\min \left\{U_{m}, V_{n}\right\}\right)^{\alpha}}{\left(\max \left\{U_{m}, V_{n}\right\}\right)^{\lambda+\alpha}} \frac{V_{n}^{\lambda_{2}} U^{\prime}(x)}{U_{m}^{1-\lambda_{1}}} d x \\
&>\sum_{m=m_{0}}^{\infty} \int_{m}^{m+1} \frac{\left(\min \left\{U(x), V_{n}\right\}\right)^{\alpha}}{\left(\max \left\{U(x), V_{n}\right\}\right)^{\lambda+\alpha}} \frac{V_{n}^{\lambda_{2}} U^{\prime}(x)}{U^{1-\lambda_{1}}(x)} d x \\
& \stackrel{t=}{\stackrel{U(x) / V_{n}}{=}} \sum_{m=m_{0}}^{\infty} \int_{\frac{U(m)}{V_{n}}}^{\frac{U(m+1)}{V_{n}}} \frac{(\min \{t, 1\})^{\alpha}}{(\max \{t, 1\})^{\lambda+\alpha}} t^{\lambda_{1}-1} d t \\
&=\int_{\frac{U\left(m_{0}\right)}{V_{n}}}^{\infty} \frac{(\min \{t, 1\})^{\alpha}}{(\max \{t, 1\})^{\lambda+\alpha}} t^{\lambda_{1}-1} d t=k\left(\lambda_{1}\right)\left(1-\vartheta\left(\lambda_{1}, n\right)\right),
\end{aligned}
$$


where

$$
\vartheta\left(\lambda_{1}, n\right):=\frac{1}{k\left(\lambda_{1}\right)} \int_{0}^{\frac{U\left(m_{0}\right)}{V_{n}}} \frac{(\min \{t, 1\})^{\alpha}}{(\max \{t, 1\})^{\lambda+\alpha}} t^{\lambda_{1}-1} d t \in(0,1) .
$$

For $V_{n}>U\left(m_{0}\right)$, we obtain

$$
\vartheta\left(\lambda_{1}, n\right)=\frac{1}{k\left(\lambda_{1}\right)} \int_{0}^{\frac{U\left(m_{0}\right)}{V_{n}}} t^{\lambda_{1}+\alpha-1} d t=\frac{1}{\left(\lambda_{1}+\alpha\right) k\left(\lambda_{1}\right)}\left(\frac{U\left(m_{0}\right)}{V_{n}}\right)^{\lambda_{1}+\alpha} .
$$

Hence, we have (18).

For $a>0$, we find

$$
\begin{aligned}
\sum_{m=1}^{\infty} \frac{\mu_{m}}{U_{m}^{1+a}} & =\sum_{m=1}^{m_{0}} \frac{\mu_{m}}{U_{m}^{1+a}}+\sum_{m=m_{0}+1}^{\infty} \frac{\mu_{m}}{U_{m}^{1+a}} \\
& =\sum_{m=1}^{m_{0}} \frac{\mu_{m}}{U_{m}^{1+a}}+\sum_{m=m_{0}+1}^{\infty} \int_{m-1}^{m} \frac{U^{\prime}(x)}{U_{m}^{1+a}} d x \\
& <\sum_{m=1}^{m_{0}} \frac{\mu_{m}}{U_{m}^{1+a}}+\sum_{m=m_{0}+1}^{\infty} \int_{m-1}^{m} \frac{U^{\prime}(x)}{U^{1+a}(x)} d x \\
& =\sum_{m=1}^{m_{0}} \frac{\mu_{m}}{U_{m}^{1+a}}+\int_{m_{0}}^{\infty} \frac{d U(x)}{U^{1+a}(x)}=\sum_{m=1}^{m_{0}} \frac{\mu_{m}}{U_{m}^{1+a}}+\frac{1}{a U_{m_{0}}^{a}}=\frac{1}{a}\left(\frac{1}{U_{m_{0}}^{a}}+a \sum_{m=1}^{m_{0}} \frac{\mu_{m}}{U_{m}^{1+a}}\right), \\
\sum_{m=1}^{\infty} \frac{\mu_{m}}{U_{m}^{1+a}} & \geq \sum_{m=m_{0}}^{\infty} \frac{\mu_{m+1}}{U_{m}^{1+a}}=\sum_{m=m_{0}}^{\infty} \int_{m}^{m+1} \frac{U^{\prime}(x)}{U_{m}^{1+a}} d x \\
& >\sum_{m=m_{0}}^{\infty} \int_{m}^{m+1} \frac{U^{\prime}(x) d x}{U^{1+a}(x)}=\int_{m_{0}}^{\infty} \frac{d U(x)}{U^{1+a}(x)}=\frac{1}{a U_{m_{0}}^{a}} .
\end{aligned}
$$

Hence, we have (19). In the same way, have (20).

\section{Equivalent inequalities and operator expressions}

Theorem 4 If $-\alpha<\lambda_{1}, \lambda_{2} \leq 1-\alpha, \lambda_{1}+\lambda_{2}=\lambda, k\left(\lambda_{1}\right)$ is as in (9), then for $p>1,0<$ $\|a\|_{p, \Phi_{\lambda}},\|b\|_{q, \Psi_{\lambda}}<\infty$, we have the following equivalent inequalities:

$$
\begin{aligned}
& I:=\sum_{n=1}^{\infty} \sum_{m=1}^{\infty} \frac{\left(\min \left\{U_{m}, V_{n}\right\}\right)^{\alpha} a_{m} b_{n}}{\left(\max \left\{U_{m}, V_{n}\right\}\right)^{\lambda+\alpha}}<k\left(\lambda_{1}\right)\|a\|_{p, \Phi_{\lambda}}\|b\|_{q, \Psi_{\lambda}}, \\
& J:=\left\{\sum_{n=1}^{\infty} \frac{v_{n}}{V_{n}^{1-p \lambda_{2}}}\left[\sum_{m=1}^{\infty} \frac{\left(\min \left\{U_{m}, V_{n}\right\}\right)^{\alpha} a_{m}}{\left(\max \left\{U_{m}, V_{n}\right\}\right)^{\lambda+\alpha}}\right]^{p}\right\}^{\frac{1}{p}}<k\left(\lambda_{1}\right)\|a\|_{p, \Phi_{\lambda}} .
\end{aligned}
$$

Proof By Hölder's inequality with weight (see [25]) we have

$$
\begin{aligned}
& {\left[\sum_{m=1}^{\infty} \frac{\left(\min \left\{U_{m}, V_{n}\right\}\right)^{\alpha} a_{m}}{\left(\max \left\{U_{m}, V_{n}\right\}\right)^{\lambda+\alpha}}\right]^{p}} \\
& \quad=\left[\sum_{m=1}^{\infty} \frac{\left(\min \left\{U_{m}, V_{n}\right\}\right)^{\alpha}}{\left(\max \left\{U_{m}, V_{n}\right\}\right)^{\lambda+\alpha}}\left(\frac{U_{m}^{\frac{1-\lambda_{1}}{q}} a_{m}}{V_{n}^{\frac{1-\lambda_{2}}{p}} \mu_{m}^{\frac{1}{q}}}\right)\left(\frac{V_{n}^{\frac{1-\lambda_{2}}{p}} \mu_{m}^{\frac{1}{q}}}{U_{m}^{\frac{1-\lambda_{1}}{q}}}\right)\right]^{p}
\end{aligned}
$$




$$
\begin{aligned}
\leq & \sum_{m=1}^{\infty} \frac{\left(\min \left\{U_{m}, V_{n}\right\}\right)^{\alpha}}{\left(\max \left\{U_{m}, V_{n}\right\}\right)^{\lambda+\alpha}}\left(\frac{U_{m}^{\left(1-\lambda_{1}\right) p / q}}{V_{n}^{1-\lambda_{2}} \mu_{m}^{p / q}} a_{m}^{p}\right) \\
& \times\left[\sum_{m=1}^{\infty} \frac{\left(\min \left\{U_{m}, V_{n}\right\}\right)^{\alpha}}{\left(\max \left\{U_{m}, V_{n}\right\}\right)^{\lambda+\alpha}} \frac{V_{n}^{\left(1-\lambda_{2}\right)(q-1)} \mu_{m}}{U_{m}^{1-\lambda_{1}}}\right]^{p-1} \\
= & \frac{V_{n}^{1-p \lambda_{2}}}{\left(\varpi\left(\lambda_{1}, n\right)\right)^{1-p} v_{n}} \sum_{m=1}^{\infty} \frac{\left(\min \left\{U_{m}, V_{n}\right\}\right)^{\alpha}}{\left(\max \left\{U_{m}, V_{n}\right\}\right)^{\lambda+\alpha}} \frac{U_{m}^{\left(1-\lambda_{1}\right)(p-1)} v_{n}}{V_{n}^{1-\lambda_{2}} \mu_{m}^{p-1}} a_{m}^{p} .
\end{aligned}
$$

In view of (14), we find

$$
\begin{aligned}
J & \leq\left(k\left(\lambda_{1}\right)\right)^{\frac{1}{q}}\left[\sum_{n=1}^{\infty} \sum_{m=1}^{\infty} \frac{\left(\min \left\{U_{m}, V_{n}\right\}\right)^{\alpha}}{\left(\max \left\{U_{m}, V_{n}\right\}\right)^{\lambda+\alpha}} \frac{U_{m}^{\left(1-\lambda_{1}\right)(p-1)} v_{n}}{V_{n}^{1-\lambda_{2}} \mu_{m}^{p-1}} a_{m}^{p}\right]^{\frac{1}{p}} \\
& =\left(k\left(\lambda_{1}\right)\right)^{\frac{1}{q}}\left[\sum_{m=1}^{\infty} \sum_{n=1}^{\infty} \frac{\left(\min \left\{U_{m}, V_{n}\right\}\right)^{\alpha}}{\left(\max \left\{U_{m}, V_{n}\right\}\right)^{\lambda+\alpha}} \frac{U_{m}^{\left(1-\lambda_{1}\right)(p-1)} v_{n}}{V_{n}^{1-\lambda_{2}} \mu_{m}^{p-1}} a_{m}^{p}\right]^{\frac{1}{p}} \\
& =\left(k\left(\lambda_{1}\right)\right)^{\frac{1}{q}}\left[\sum_{m=1}^{\infty} \omega\left(\lambda_{2}, m\right) \frac{U_{m}^{p\left(1-\lambda_{1}\right)-1}}{\mu_{m}^{p-1}} a_{m}^{p}\right]^{\frac{1}{p}} .
\end{aligned}
$$

Then by (13) we have (24).

By Hölder's inequality we have

$$
\begin{aligned}
I & =\sum_{n=1}^{\infty}\left[\frac{v_{n}^{\frac{1}{p}}}{V_{n}^{\frac{1}{p}-\lambda_{2}}} \sum_{m=1}^{\infty} \frac{\left(\min \left\{U_{m}, V_{n}\right\}\right)^{\alpha} a_{m}}{\left(\max \left\{U_{m}, V_{n}\right\}\right)^{\lambda+\alpha}}\right]\left(\frac{V_{n}^{\frac{1}{p}-\lambda_{2}}}{v_{n}^{\frac{1}{p}}} b_{n}\right) \\
& \leq J\|b\|_{q, \Psi_{\lambda} .}
\end{aligned}
$$

Then by (24) we have (23).

On the other hand, assuming that (23) is valid, we set

$$
b_{n}:=\frac{v_{n}}{V_{n}^{1-p \lambda_{2}}}\left[\sum_{m=1}^{\infty} \frac{\left(\min \left\{U_{m}, V_{n}\right\}\right)^{\alpha} a_{m}}{\left(\max \left\{U_{m}, V_{n}\right\}\right)^{\lambda+\alpha}}\right]^{p-1}, \quad n \in \mathbf{N}
$$

Then we find $J^{p}=\|b\|_{q, \Psi_{\lambda}}^{q}$. If $J=0$, then (24) is trivially valid; if $J=\infty$, then by (26) and (13) it is impossible. Suppose that $0<J<\infty$. By (23) it follows that

$$
\begin{aligned}
& \|b\|_{q, \Psi_{\lambda}}^{q}=J^{p}=I<k\left(\lambda_{1}\right)\|a\|_{p, \Phi_{\lambda}}\|b\|_{q, \Psi_{\lambda}}, \\
& \|b\|_{q, \Psi_{\lambda}}^{q-1}=J<k_{s}\left(\lambda_{1}\right)\|a\|_{p, \Phi_{\lambda}},
\end{aligned}
$$

and then (24) follows, which is equivalent to (23).

Theorem 5 With the assumptions of Theorem 4 , if $m_{0}, n_{0} \in \mathbf{N}, \mu_{m} \geq \mu_{m+1}\left(m \in\left\{m_{0}, m_{0}+\right.\right.$ $1, \ldots\}), v_{n} \geq v_{n+1}\left(n \in\left\{n_{0}, n_{0}+1, \ldots\right\}\right), U(\infty)=V(\infty)=\infty$, then the constant factor $k\left(\lambda_{1}\right)$ in (23) and (24) is the best possible. 
Proof For $\varepsilon \underset{\sim}{\in}\left(0, \underset{\sim}{p}\left(\lambda_{1}+\alpha\right)\right)$, we set $\tilde{\lambda}_{1}=\lambda_{1}-\frac{\varepsilon}{p}(\in(-\alpha, 1-\alpha)), \tilde{\lambda}_{2}=\lambda_{2}+\frac{\varepsilon}{p}(>-\alpha)$, and $\widetilde{a}=\left\{\widetilde{a}_{m}\right\}_{m=1}^{\infty}, \widetilde{b}=\left\{\widetilde{b}_{n}\right\}_{n=1}^{\infty}$,

$$
\tilde{a}_{m}:=U_{m}^{\widetilde{\lambda}_{1}-1} \mu_{m}=U_{m}^{\lambda_{1}-\frac{\varepsilon}{p}-1} \mu_{m}, \quad \widetilde{b}_{n}=V_{n}^{\widetilde{\lambda}_{2}-\varepsilon-1} v_{n}=V_{n}^{\lambda_{2}-\frac{\varepsilon}{q}-1} v_{n} .
$$

Then by (19), (20), and (18) we have

$$
\begin{aligned}
& \|\widetilde{a}\|_{p, \Phi_{\lambda}}\|\widetilde{b}\|_{q, \Psi_{\lambda}}=\left(\sum_{m=1}^{\infty} \frac{\mu_{m}}{U_{m}^{1+\varepsilon}}\right)^{\frac{1}{p}}\left(\sum_{n=1}^{\infty} \frac{v_{n}}{V_{n}^{1+\varepsilon}}\right)^{\frac{1}{q}} \\
& \quad=\frac{1}{\varepsilon}\left(\frac{1}{U_{m_{0}}^{\varepsilon}}+\varepsilon O(1)\right)^{\frac{1}{p}}\left(\frac{1}{V_{n_{0}}^{\varepsilon}}+\varepsilon \widetilde{O}(1)\right)^{\frac{1}{q}}, \\
& \widetilde{I}:=\sum_{n=1}^{\infty} \sum_{m=1}^{\infty} \frac{\left(\min \left\{U_{m}, V_{n}\right\}\right)^{\alpha}}{\left(\max \left\{U_{m}, V_{n}\right\}\right)^{\lambda+\alpha}} \widetilde{a}_{m} \widetilde{b}_{n} \\
& =\sum_{n=1}^{\infty}\left[\sum_{m=1}^{\infty} \frac{\left(\min \left\{U_{m}, V_{n}\right\}\right)^{\alpha}}{\left(\max \left\{U_{m}, V_{n}\right\}\right)^{\lambda+\alpha}} \frac{V_{n}^{\tilde{\lambda}_{2}} \mu_{m}}{U_{m}^{1-\widetilde{\lambda}_{1}}}\right] \frac{v_{n}}{V_{n}^{\varepsilon+1}} \\
& =\sum_{n=1}^{\infty} \varpi\left(\tilde{\lambda}_{1}, n\right) \frac{v_{n}}{V_{n}^{\varepsilon+1}} \geq k\left(\tilde{\lambda}_{1}\right) \sum_{n=1}^{\infty}\left(1-\vartheta\left(\tilde{\lambda}_{1}, n\right)\right) \frac{v_{n}}{V_{n}^{\varepsilon+1}} \\
& =k\left(\tilde{\lambda}_{1}\right)\left(\sum_{n=1}^{\infty} \frac{v_{n}}{V_{n}^{\varepsilon+1}}-\sum_{n=1}^{\infty} O\left(\frac{v_{n}}{V_{n}^{\frac{\varepsilon}{q}+\lambda_{1}+\alpha+1}}\right)\right) \\
& =\frac{1}{\varepsilon} k\left(\widetilde{\lambda}_{1}\right)\left[\frac{1}{V_{n_{0}}^{\varepsilon}}+\varepsilon(\widetilde{O}(1)-O(1))\right] .
\end{aligned}
$$

If there exists a positive constant $K \leq k\left(\lambda_{1}\right)$ such that (23) is valid when replacing $k\left(\lambda_{1}\right)$ to $K$, then, in particular, we have $\varepsilon \widetilde{I}<\varepsilon K\|\widetilde{a}\|_{p, \Phi_{\lambda}}\|\widetilde{b}\|_{q, \Psi_{\lambda}}$, namely,

$$
k\left(\widetilde{\lambda}_{1}\right)\left[\frac{1}{V_{n_{0}}^{\varepsilon}}+\varepsilon(\widetilde{O}(1)-O(1))\right]<K\left(\frac{1}{U_{m_{0}}^{\varepsilon}}+\varepsilon O(1)\right)^{\frac{1}{p}}\left(\frac{1}{V_{n_{0}}^{\varepsilon}}+\varepsilon \widetilde{O}(1)\right)^{\frac{1}{q}} .
$$

It follows that $k\left(\lambda_{1}\right) \leq K\left(\varepsilon \rightarrow 0^{+}\right)$. Hence, $K=k\left(\lambda_{1}\right)$ is the best possible constant factor of (23).

The constant factor $k\left(\lambda_{1}\right)$ in (24) is still the best possible. Otherwise, we would reach a contradiction by (27) that the constant factor in (23) is not the best possible.

For $p>1$, we find $\Psi_{\lambda}^{1-p}(n)=\frac{v_{n}}{V_{n}^{1-p \lambda_{2}}}$ and define the following normed spaces:

$$
\begin{aligned}
& l_{p, \Phi_{\lambda}}:=\left\{a=\left\{a_{m}\right\}_{m=1}^{\infty} ;\|a\|_{p, \Phi_{\lambda}}<\infty\right\}, \\
& l_{q, \Psi_{\lambda}}:=\left\{b=\left\{b_{n}\right\}_{n=1}^{\infty} ;\|b\|_{q, \Psi_{\lambda}}<\infty\right\}, \\
& l_{p, \Psi_{\lambda}^{1-p}}:=\left\{c=\left\{c_{n}\right\}_{n=1}^{\infty} ;\|c\|_{\left.p, \Psi_{\lambda}^{1-p}<\infty\right\} .}\right.
\end{aligned}
$$

Assuming that $a=\left\{a_{m}\right\}_{m=1}^{\infty} \in l_{p, \Phi_{\lambda}}$ and setting

$$
c=\left\{c_{n}\right\}_{n=1}^{\infty}, \quad c_{n}:=\sum_{m=1}^{\infty} \frac{\left(\min \left\{U_{m}, V_{n}\right\}\right)^{\alpha}}{\left(\max \left\{U_{m}, V_{n}\right\}\right)^{\lambda+\alpha}} a_{m}, \quad n \in \mathbf{N},
$$


we can rewrite $(24)$ as

$$
\|c\|_{p, \Psi_{\lambda}^{1-p}}<k\left(\lambda_{1}\right)\|a\|_{p, \Phi_{\lambda}}<\infty
$$

namely, $c \in l_{p, \Psi_{\lambda}^{1-p}}$.

Definition 1 Define a Hardy-Hilbert-type operator $T: l_{p, \Phi_{\lambda}} \rightarrow l_{p, \Psi_{\lambda}^{1-p}}$ as follows: For any $a=\left\{a_{m}\right\}_{m=1}^{\infty} \in l_{p, \Phi_{\lambda}}$, there exists a unique representation $T a=c \in l_{p, \Psi_{\lambda}^{1-p}}$. Define the formal inner product of $T a$ and $b=\left\{b_{n}\right\}_{n=1}^{\infty} \in l_{q, \Psi_{\lambda}}$ as follows:

$$
(T a, b):=\sum_{n=1}^{\infty}\left[\sum_{m=1}^{\infty} \frac{\left(\min \left\{U_{m}, V_{n}\right\}\right)^{\alpha}}{\left(\max \left\{U_{m}, V_{n}\right\}\right)^{\lambda+\alpha}} a_{m}\right] b_{n} .
$$

Then we can rewrite (23) and (24) as follows:

$$
\begin{aligned}
& (T a, b)<k\left(\lambda_{1}\right)\|a\|_{p, \Phi_{\lambda}}\|b\|_{q, \Psi_{\lambda}}, \\
& \|T a\|_{p, \Psi_{\lambda}^{1-p}}<k\left(\lambda_{1}\right)\|a\|_{p, \Phi_{\lambda}} .
\end{aligned}
$$

Define the norm of the operator $T$ as follows:

$$
\|T\|:=\sup _{a(\neq \theta) \in l_{p, \Phi_{\lambda}}} \frac{\|T a\|_{p, \Psi_{\lambda}^{1-p}}}{\|a\|_{p, \Phi_{\lambda}}} .
$$

Then by (31) we find $\|T\| \leq k\left(\lambda_{1}\right)$. Since by Theorem 5 the constant factor in (31) is the best possible, we have

$$
\|T\|=k\left(\lambda_{1}\right)=\frac{\lambda+2 \alpha}{\left(\lambda_{1}+\alpha\right)\left(\lambda_{2}+\alpha\right)} .
$$

\section{Some equivalent reverse inequalities}

Theorem 6 If $-\alpha<\lambda_{1}, \lambda_{2} \leq 1-\alpha, \lambda_{1}+\lambda_{2}=\lambda, k\left(\lambda_{1}\right)$ is as in (9), $m_{0}, n_{0} \in \mathbf{N}, \mu_{m} \geq \mu_{m+1}$ $\left(m \in\left\{m_{0}, m_{0}+1, \ldots\right\}\right), v_{n} \geq v_{n+1}\left(n \in\left\{n_{0}, n_{0}+1, \ldots\right\}\right), U(\infty)=V(\infty)=\infty$, then for $0<$ $p<1,0<\|a\|_{p, \Phi_{\lambda}},\|b\|_{q, \Psi_{\lambda}}<\infty$, we have the following equivalent inequalities with the best possible constant factor $k\left(\lambda_{1}\right)$ :

$$
\begin{aligned}
& I=\sum_{n=1}^{\infty} \sum_{m=1}^{\infty} \frac{\left(\min \left\{U_{m}, V_{n}\right\}\right)^{\alpha}}{\left(\max \left\{U_{m}, V_{n}\right\}\right)^{\lambda+\alpha}} a_{m} b_{n}>k\left(\lambda_{1}\right)\|a\|_{p, \widetilde{\Phi}_{\lambda}}\|b\|_{q, \Psi_{\lambda},} \\
& J=\left\{\sum_{n=1}^{\infty} \frac{v_{n}}{V_{n}^{1-p \lambda_{2}}}\left[\sum_{m=1}^{\infty} \frac{\left(\min \left\{U_{m}, V_{n}\right\}\right)^{\alpha} a_{m}}{\left(\max \left\{U_{m}, V_{n}\right\}\right)^{\lambda+\alpha}}\right]^{p}\right\}^{\frac{1}{p}}>k\left(\lambda_{1}\right)\|a\|_{p, \widetilde{\Phi}_{\lambda}} .
\end{aligned}
$$

Proof By the reverse Hölder's inequality and (14), we have the reverses of (25), (26), and (27). Then by (17) we have (35). By (35) and the reverse of (27) we have (34).

On the other hand, assuming that (34) is valid, we set $b_{n}$ as in Theorem 4 . Then we find $J^{p}=\|b\|_{q, \Psi_{\lambda}}^{q}$. If $J=\infty$, then (35) is trivially valid; if $J=0$, then by reverse of (26) and (17) it 
is impossible. Suppose that $0<J<\infty$. By (34) it follows that

$$
\begin{aligned}
& \|b\|_{q, \Psi_{\lambda}}^{q}=J^{p}=I>k_{s}\left(\lambda_{1}\right)\|a\|_{p, \widetilde{\Phi}_{\lambda}}\|b\|_{q, \Psi_{\lambda},}, \\
& \|b\|_{q, \Psi_{\lambda}}^{q-1}=J>k_{s}\left(\lambda_{1}\right)\|a\|_{p, \widetilde{\Phi}_{\lambda},}
\end{aligned}
$$

and then (35) follows, which is equivalent to (34).

For $\varepsilon \in\left(0, p\left(\lambda_{1}+\alpha\right)\right)$, we set $\tilde{\lambda}_{1}, \widetilde{\lambda}_{2}, \widetilde{a}_{m}$, and $\widetilde{b}_{n}$ as (30). Then by (19), (20), and (14) we find

$$
\begin{aligned}
\|a\|_{p, \widetilde{\Phi}_{\lambda}}\|b\|_{q, \Psi_{\lambda}} & =\left[\sum_{m=1}^{\infty}\left(1-\theta\left(\lambda_{2}, m\right)\right) \frac{\mu_{m}}{U_{m}^{1+\varepsilon}}\right]^{\frac{1}{p}}\left(\sum_{n=1}^{\infty} \frac{v_{n}}{V_{n}^{1+\varepsilon}}\right)^{\frac{1}{q}} \\
& =\left(\sum_{m=1}^{\infty} \frac{\mu_{m}}{U_{m}^{1+\varepsilon}}-\sum_{m=1}^{\infty} O\left(\frac{\mu_{m}}{U_{m}^{1+\lambda_{2}+\alpha+\varepsilon}}\right)\right)^{\frac{1}{p}}\left(\sum_{n=1}^{\infty} \frac{v_{n}}{V_{n}^{1+\varepsilon}}\right)^{\frac{1}{q}} \\
& =\frac{1}{\varepsilon}\left[\frac{1}{U_{m}^{\varepsilon}}+\varepsilon\left(O(1)-O_{1}(1)\right)\right]^{\frac{1}{p}}\left(\frac{1}{V_{n_{0}}^{\varepsilon}}+\varepsilon \widetilde{O}(1)\right)^{\frac{1}{q}} \\
\widetilde{I} & =\sum_{n=1}^{\infty} \sum_{m=1}^{\infty} \frac{\left(\min \left\{U_{m}, V_{n}\right\}\right)^{\alpha}}{\left(\max \left\{U_{m}, V_{n}\right\}\right)^{\lambda+\alpha}} \widetilde{a}_{m} \widetilde{b}_{n} \\
& =\sum_{n=1}^{\infty}\left[\sum_{m=1}^{\infty} \frac{\left(\min \left\{U_{m}, V_{n}\right\}\right)^{\alpha}}{\left(\max \left\{U_{m}, V_{n}\right\}\right)^{\lambda+\alpha}} \frac{V_{n}^{\tilde{\lambda}_{2}} \mu_{m}}{U_{m}^{1-\widetilde{\lambda}_{1}}}\right] \frac{v_{n}}{V_{n}^{\varepsilon+1}} \\
& =\sum_{n=1}^{\infty} \varpi\left(\widetilde{\lambda}_{1}, n\right) \frac{v_{n}}{V_{n}^{\varepsilon+1}} \leq k\left(\widetilde{\lambda}_{1}\right) \sum_{n=1}^{\infty} \frac{v_{n}}{V_{n}^{\varepsilon+1}} \\
& =\frac{1}{\varepsilon} k\left(\widetilde{\lambda}_{1}\right)\left(\frac{1}{V_{n_{0}}^{\varepsilon}}+\varepsilon \widetilde{O}(1)\right) .
\end{aligned}
$$

If there exists a constant $K \geq k\left(\lambda_{1}\right)$ such that (34) is valid when replacing $k\left(\lambda_{1}\right)$ to $K$, then, in particular, we have $\varepsilon \widetilde{I}>\varepsilon K\|\widetilde{a}\|_{p, \widetilde{\Phi}_{\lambda}}\|\widetilde{b}\|_{q, \Psi_{\lambda}}$, namely,

$$
k\left(\widetilde{\lambda}_{1}\right)\left(\frac{1}{V_{n_{0}}^{\varepsilon}}+\varepsilon \widetilde{O}(1)\right)>K\left[\frac{1}{U_{m_{0}}^{\varepsilon}}+\varepsilon\left(O(1)-O_{1}(1)\right)\right]^{\frac{1}{p}}\left(\frac{1}{V_{n_{0}}^{\varepsilon}}+\varepsilon \widetilde{O}(1)\right)^{\frac{1}{q}} .
$$

It follows that $k\left(\lambda_{1}\right) \geq K\left(\varepsilon \rightarrow 0^{+}\right)$. Hence, $K=k\left(\lambda_{1}\right)$ is the best possible constant factor of (34).

The constant factor $k\left(\lambda_{1}\right)$ in (35) is still the best possible. Otherwise, we would reach a contradiction by the reverse of (27) that the constant factor in (34) is not the best possible.

Theorem 7 With the assumptions of Theorem 6, if $p<0$, then we have the following equivalent inequalities with the best possible constant factor $k\left(\lambda_{1}\right)$ :

$$
I=\sum_{n=1}^{\infty} \sum_{m=1}^{\infty} \frac{\left(\min \left\{U_{m}, V_{n}\right\}\right)^{\alpha}}{\left(\max \left\{U_{m}, V_{n}\right\}\right)^{\lambda+\alpha}} a_{m} b_{n}>k\left(\lambda_{1}\right)\|a\|_{p, \Phi_{\lambda}}\|b\|_{q, \widetilde{\Psi}_{\lambda}}
$$




$$
\begin{aligned}
J_{1} & :=\left\{\sum_{n=1}^{\infty} \frac{V_{n}^{p \lambda_{2}-1} v_{n}}{\left(1-\vartheta\left(\lambda_{1}, n\right)\right)^{p-1}}\left[\sum_{m=1}^{\infty} \frac{\left(\min \left\{U_{m}, V_{n}\right\}\right)^{\alpha} a_{m}}{\left(\max \left\{U_{m}, V_{n}\right\}\right)^{\lambda+\alpha}}\right]^{p}\right\}^{\frac{1}{p}} \\
& >k\left(\lambda_{1}\right)\|a\|_{p, \Phi_{\lambda}} .
\end{aligned}
$$

Proof By the reverse Hölder inequality with weight, since $p<0$, by (18) we have

$$
\begin{aligned}
& {\left[\sum_{m=1}^{\infty} \frac{\left(\min \left\{U_{m}, V_{n}\right\}\right)^{\alpha}}{\left(\max \left\{U_{m}, V_{n}\right\}\right)^{\lambda+\alpha}} a_{m}\right]^{p} } {\left[\sum_{m=1}^{\infty} \frac{\left(\min \left\{U_{m}, V_{n}\right\}\right)^{\alpha}}{\left(\max \left\{U_{m}, V_{n}\right\}\right)^{\lambda+\alpha}}\left(\frac{U_{m}^{\left(1-\lambda_{1}\right) / q}}{V_{n}^{\left(1-\lambda_{2}\right) / p} \mu_{m}^{1 / q}} a_{m}\right)\left(\frac{V_{n}^{\left(1-\lambda_{2}\right) / p} \mu_{m}^{1 / q}}{U_{m}^{\left(1-\lambda_{1}\right) / q}}\right)\right]^{p} } \\
& \leq \sum_{m=1}^{\infty} \frac{\left(\min \left\{U_{m}, V_{n}\right\}\right)^{\alpha}}{\left(\max \left\{U_{m}, V_{n}\right\}\right)^{\lambda+\alpha}} \frac{U_{m}^{\left(1-\lambda_{1}\right) p / q}}{V_{n}^{1-\lambda_{2}} \mu_{m}^{p / q}} a_{m}^{p} \\
& \times\left[\sum_{m=1}^{\infty} \frac{\left(\min \left\{U_{m}, V_{n}\right\}\right)^{\alpha}}{\left(\max \left\{U_{m}, V_{n}\right\}\right)^{\lambda+\alpha}} \frac{V_{n}^{\left(1-\lambda_{2}\right)(q-1)} \mu_{m}}{U_{m}^{1-\lambda_{1}}}\right]^{p-1} \\
&= \frac{V_{n}^{1-p \lambda_{2}}}{\left(\varpi\left(\lambda_{1}, n\right)\right)^{1-p}} \sum_{m=1}^{\infty} \frac{\left(\min \left\{U_{m}, V_{n}\right\}\right)^{\alpha}}{\left(\max \left\{U_{m}, V_{n}\right\}\right)^{\lambda+\alpha}} \frac{U_{m}^{\left(1-\lambda_{1}\right)(p-1)}}{V_{n}^{1-\lambda_{2}} \mu_{m}^{p-1}} a_{m}^{p} \\
& \leq \frac{\left(k\left(\lambda_{1}\right)\right)^{p-1} V_{n}^{1-p \lambda_{2}}}{\left(1-\vartheta\left(\lambda_{1}, n\right)\right)^{1-p} v_{n}} \sum_{m=1}^{\infty} \frac{\left(\min \left\{U_{m}, V_{n}\right\}\right)^{\alpha}}{\left(\max \left\{U_{m}, V_{n}\right\}\right)^{\lambda+\alpha}} \frac{U_{m}^{\left(1-\lambda_{1}\right)(p-1)} v_{n}}{V_{n}^{1-\lambda_{2}} \mu_{m}^{p-1}} a_{m}^{p}, \\
& J_{1} \geq\left(k\left(\lambda_{1}\right)\right)^{\frac{1}{q}}\left\{\sum_{n=1}^{\infty} \sum_{m=1}^{\infty} \frac{\left(\min \left\{U_{m}, V_{n}\right\}\right)^{\alpha}}{\left(\max \left\{U_{m}, V_{n}\right\}\right)^{\lambda+\alpha}} \frac{U_{m}^{\left(1-\lambda_{1}\right)(p-1)} v_{n}}{V_{n}^{1-\lambda_{2}} \mu_{m}^{p-1}} a_{m}^{p}\right\} \\
&=\left(k\left(\lambda_{1}\right)\right)^{\frac{1}{q}}\left\{\sum_{m=1}^{\infty} \sum_{n=1}^{\infty} \frac{\left(\min \left\{U_{m}, V_{n}\right\}\right)^{\alpha}}{\left(\max \left\{U_{m}, V_{n}\right\}\right)^{\lambda+\alpha}} a_{m} \frac{U_{m}^{\left(1-\lambda_{1}\right)(p-1)} v_{n}}{V_{n}^{1-\lambda_{2}} \mu_{m}^{p-1}} a_{m}^{p}\right\} \\
&=\left(k\left(\lambda_{1}\right)\right)^{\frac{1}{q}}\left\{\sum_{m=1}^{\infty} \omega\left(\lambda_{2}, m\right) \frac{U_{m}^{p\left(1-\lambda_{1}\right)-1}}{\mu_{m}^{p-1}} a_{m}^{p}\right\} \\
& \frac{1}{p}
\end{aligned}
$$

Then by (13) we have (39).

By the reverse Hölder inequality we have

$$
\begin{aligned}
I= & \sum_{n=1}^{\infty} \frac{V_{n}^{\lambda_{2}-\frac{1}{p}} v_{n}^{1 / p}}{\left(1-\vartheta\left(\lambda_{1}, n\right)\right)^{1 / q}}\left[\sum_{m=1}^{\infty} \frac{\left(\min \left\{U_{m}, V_{n}\right\}\right)^{\alpha}}{\left(\max \left\{U_{m}, V_{n}\right\}\right)^{\lambda+\alpha}} a_{m}\right] \\
& \times\left[\left(1-\vartheta\left(\lambda_{1}, n\right)\right)^{\frac{1}{q}} \frac{V_{n}^{\frac{1}{p}-\lambda_{2}}}{v_{n}^{1 / p}} b_{n}\right] \geq J_{1}\|b\|_{q, \widetilde{\Psi}_{\lambda}} .
\end{aligned}
$$

Then by (39) we have (38).

On the other hand, assuming that (38) is valid, we set $b_{n}$ as follows:

$$
b_{n}:=\frac{V_{n}^{p \lambda_{2}-1} v_{n}}{\left(1-\vartheta\left(\lambda_{1}, n\right)\right)^{p-1}}\left[\sum_{m=1}^{\infty} \frac{\left(\min \left\{U_{m}, V_{n}\right\}\right)^{\alpha}}{\left(\max \left\{U_{m}, V_{n}\right\}\right)^{\lambda+\alpha}} a_{m}\right]^{p-1}, \quad n \in \mathbf{N} .
$$


Then we find $J_{1}^{p}=\|b\|_{q, \widetilde{\Psi}_{\lambda}}^{q}$. If $J_{1}=\infty$, then (39) is trivially valid; if $J_{1}=0$, then by (40) and (13) it is impossible. Suppose that $0<J_{1}<\infty$. By (38) it follows that

$$
\begin{aligned}
& \|b\|_{q, \widetilde{\Psi}_{\lambda}}^{q}=J_{1}^{p}=I>k_{s}\left(\lambda_{1}\right)\|a\|_{p, \Phi_{\lambda}}\|b\|_{q, \widetilde{\Psi}_{\lambda}}, \\
& \|b\|_{q, \widetilde{\Psi}_{\lambda}}^{q-1}=J_{1}>k_{s}\left(\lambda_{1}\right)\|a\|_{p, \Phi_{\lambda}},
\end{aligned}
$$

and then (39) follows, which is equivalent to (38).

For $\varepsilon \in\left(0, q\left(\lambda_{2}+\alpha\right)\right)$, we set $\tilde{\lambda}_{1}=\lambda_{1}+\frac{\varepsilon}{q}(>-\alpha), \tilde{\lambda}_{2}=\lambda_{2}-\frac{\varepsilon}{q}(\in(-\alpha, 1-\alpha))$, and

$$
\tilde{a}_{m}:=U_{m}^{\widetilde{\lambda}_{1}-1-\varepsilon} \mu_{m}=U_{m}^{\lambda_{1}-\frac{\varepsilon}{p}-1} \mu_{m}, \quad \widetilde{b}_{n}=V_{n}^{\tilde{\lambda}_{2}-1} v_{n}=V_{n}^{\lambda_{2}-\frac{\varepsilon}{q}-1} v_{n} .
$$

Then by (19), (20), and (13) we have

$$
\begin{aligned}
\|\widetilde{a}\|_{p, \Phi_{\lambda}}\|\widetilde{b}\|_{q, \widetilde{\Psi}_{\lambda}} & =\left(\sum_{m=1}^{\infty} \frac{\mu_{m}}{U_{m}^{1+\varepsilon}}\right)^{\frac{1}{p}}\left[\sum_{n=1}^{\infty}\left(1-\vartheta\left(\lambda_{1}, n\right)\right) \frac{v_{n}}{V_{n}^{1+\varepsilon}}\right]^{\frac{1}{q}} \\
& =\left(\sum_{m=1}^{\infty} \frac{\mu_{m}}{U_{m}^{1+\varepsilon}}\right)^{\frac{1}{p}}\left(\sum_{n=1}^{\infty} \frac{v_{n}}{V_{n}^{1+\varepsilon}}-\sum_{n=1}^{\infty} O\left(\frac{v_{n}}{V_{n}^{1+\lambda_{1}+\alpha+\varepsilon}}\right)\right)^{\frac{1}{q}} \\
& =\frac{1}{\varepsilon}\left(\frac{1}{U_{m_{0}}^{\varepsilon}}+\varepsilon O(1)\right)^{\frac{1}{p}}\left[\frac{1}{V_{n_{0}}^{\varepsilon}}+\varepsilon\left(\widetilde{O}(1)-O_{1}(1)\right)\right]^{\frac{1}{q}} \\
\widetilde{I} & =\sum_{n=1}^{\infty} \sum_{m=1}^{\infty} \frac{\left(\min \left\{U_{m}, V_{n}\right\}\right)^{\alpha}}{\left(\max \left\{U_{m}, V_{n}\right\}\right)^{\lambda+\alpha}} \widetilde{a}_{m} \widetilde{b}_{n} \\
& =\sum_{m=1}^{\infty}\left[\sum_{n=1}^{\infty} \frac{\left(\min \left\{U_{m}, V_{n}\right\}\right)^{\alpha}}{\left(\max \left\{U_{m}, V_{n}\right\}\right)^{\lambda+\alpha}} \frac{U_{m}^{\tilde{\lambda}_{1}} U_{n}}{V_{n}^{1-\tilde{\lambda}_{2}}}\right] \frac{\mu_{m}}{U_{m}^{1+\varepsilon}} \\
& =\sum_{m=1}^{\infty} \omega\left(\widetilde{\lambda}_{2}, m\right) \frac{\mu_{m}}{U_{m}^{1+\varepsilon}} \leq k\left(\widetilde{\lambda}_{1}\right) \sum_{n=1}^{\infty} \frac{\mu_{m}}{U_{m}^{1+\varepsilon}} \\
& =\frac{1}{\varepsilon} k\left(\widetilde{\lambda}_{1}\right)\left(\frac{1}{U_{m_{0}}^{\varepsilon}}+\varepsilon O(1)\right) .
\end{aligned}
$$

If there exists a constant $K \geq k\left(\lambda_{1}\right)$ such that (38) is valid when replacing $k\left(\lambda_{1}\right)$ to $K$, then, in particular, we have $\varepsilon \widetilde{I}>\varepsilon K\|\widetilde{a}\|_{p, \Phi_{\lambda}}\|\widetilde{b}\|_{q, \widetilde{\Psi}_{\lambda}}$, namely,

$$
k\left(\widetilde{\lambda}_{1}\right)\left(\frac{1}{U_{m_{0}}^{\varepsilon}}+\varepsilon O(1)\right)>K\left(\frac{1}{U_{m_{0}}^{\varepsilon}}+\varepsilon O(1)\right)^{\frac{1}{p}}\left[\frac{1}{V_{n_{0}}^{\varepsilon}}+\varepsilon\left(\widetilde{O}(1)-O_{1}(1)\right)\right]^{\frac{1}{q}} .
$$

It follows that $k\left(\lambda_{1}\right) \geq K\left(\varepsilon \rightarrow 0^{+}\right)$. Hence, $K=k\left(\lambda_{1}\right)$ is the best possible constant factor of (38).

The constant factor $k\left(\lambda_{1}\right)$ in (39) is still the best possible. Otherwise, we would reach a contradiction by (41) that the constant factor in (38) is not the best possible. 
Remark 1 (i) For $\alpha=0$ and $0<\lambda_{1}, \lambda_{2} \leq 1$ in (23) and (24), we have the following equivalent inequalities:

$$
\begin{aligned}
& \sum_{n=1}^{\infty} \sum_{m=1}^{\infty} \frac{a_{m} b_{n}}{\left(\max \left\{U_{m}, V_{n}\right\}\right)^{\lambda}}<\frac{\lambda}{\lambda_{1} \lambda_{2}}\|a\|_{p, \Phi_{\lambda}}\|b\|_{q, \Psi_{\lambda}}, \\
& \left\{\sum_{n=1}^{\infty} \frac{v_{n}}{V_{n}^{1-p \lambda_{2}}}\left[\sum_{m=1}^{\infty} \frac{a_{m}}{\left(\max \left\{U_{m}, V_{n}\right\}\right)^{\lambda}}\right]^{p}\right\}^{\frac{1}{p}}<\frac{\lambda}{\lambda_{1} \lambda_{2}}\|a\|_{p, \Phi_{\lambda}} ;
\end{aligned}
$$

(ii) for $\alpha=-\lambda$ and $-1 \leq \lambda_{1}, \lambda_{2}<0$ in (23) and (24), we have the following equivalent inequalities:

$$
\begin{aligned}
& \sum_{n=1}^{\infty} \sum_{m=1}^{\infty} \frac{a_{m} b_{n}}{\left(\min \left\{U_{m}, V_{n}\right\}\right)^{\lambda}}<\frac{(-\lambda)}{\lambda_{1} \lambda_{2}}\|a\|_{p, \Phi_{\lambda}}\|b\|_{q, \Psi_{\lambda},} \\
& \left\{\sum_{n=1}^{\infty} \frac{v_{n}}{V_{n}^{1-p \lambda_{2}}}\left[\sum_{m=1}^{\infty} \frac{a_{m}}{\left(\min \left\{U_{m}, V_{n}\right\}\right)^{\lambda}}\right]^{p}\right\}^{\frac{1}{p}}<\frac{(-\lambda)}{\lambda_{1} \lambda_{2}}\|a\|_{p, \Phi_{\lambda}} ;
\end{aligned}
$$

(iii) for $\lambda=0,\left|\lambda_{1}\right|<\alpha\left(0<\alpha \leq \frac{1}{2}\right)$; $\left|\lambda_{1}\right|<1-\alpha\left(\frac{1}{2}<\alpha \leq 1\right), \lambda_{2}=-\lambda_{1}$ in (23) and (24), we have the following equivalent inequalities:

$$
\begin{aligned}
& \sum_{n=1}^{\infty} \sum_{m=1}^{\infty}\left(\frac{\min \left\{U_{m}, V_{n}\right\}}{\max \left\{U_{m}, V_{n}\right\}}\right)^{\alpha} a_{m} b_{n}<\frac{2 \alpha}{\alpha^{2}-\lambda_{1}^{2}}\|a\|_{p, \Phi_{\lambda}}\|b\|_{q, \Psi_{\lambda}}, \\
& \left\{\sum_{n=1}^{\infty} \frac{v_{n}}{V_{n}^{1+p \lambda_{1}}}\left[\sum_{m=1}^{\infty}\left(\frac{\min \left\{U_{m}, V_{n}\right\}}{\max \left\{U_{m}, V_{n}\right\}}\right)^{\alpha} a_{m}\right]^{p}\right\}^{\frac{1}{p}}<\frac{2 \alpha}{\alpha^{2}-\lambda_{1}^{2}}\|a\|_{p, \Phi_{\lambda}} .
\end{aligned}
$$

In view of Theorem 5, the constant factors in these inequalities with the particular kernels are all the best possible.

\section{Competing interests}

The authors declare that they have no competing interests

\section{Authors' contributions}

BY carried out the mathematical studies, participated in the sequence alignment, and drafted the manuscript. YS participated in the design of the study and performed the numerical analysis. All authors read and approved the final manuscript.

\section{Author details}

${ }^{1}$ Normal College of Jishou University, Jishou, Hunan 416000, P.R. China. ${ }^{2}$ Department of Mathematics, Guangdong University of Education, Guangzhou, Guangdong 51003, P.R. China.

\section{Acknowledgements}

This work is supported by Hunan Province Natural Science Foundation (No. 2015JJ4041) and Science Research General Foundation Item of Hunan Institution of Higher Learning College and University (No. 14C0938).

Received: 11 May 2015 Accepted: 21 November 2015 Published online: 03 December 2015

\section{References}

1. Hardy, GH, Littlewood, JE, Pólya, G: Inequalities. Cambridge University Press, Cambridge (1934)

2. Mitrinović, DS, Pečarić, JE, Fink, AM: Inequalities Involving Functions and Their Integrals and Derivatives. Kluwer Academic, Boston (1991)

3. Yang, BC: Hilbert-Type Integral Inequalities. Bentham Science Publishers, Sharjah (2009)

4. Yang, BC: Discrete Hilbert-Type Inequalities. Bentham Science Publishers, Sharjah (2011)

5. Yang, BC: The Norm of Operator and Hilbert-Type Inequalities. Science Press, Beijing (2009) 
6. Yang, BC: On Hilbert's integral inequality. J. Math. Anal. Appl. 220, 778-785 (1998)

7. Yang, BC, Brnetić, I, Krnić, M, Pečarić, JE: Generalization of Hilbert and Hardy-Hilbert integral inequalities. Math. Inequal. Appl. 8(2), 259-272 (2005)

8. Krnić, M, Pečarić, JE: Hilbert's inequalities and their reverses. Publ. Math. (Debr.) 67(3-4), 315-331 (2005)

9. Yang, BC, Rassias, TM: On the way of weight coefficient and research for Hilbert-type inequalities. Math. Inequal. Appl. 6(4), 625-658 (2003)

10. Yang, BC, Rassias, TM: On a Hilbert-type integral inequality in the subinterval and its operator expression. Banach J. Math. Anal. 4(2), 100-110 (2010)

11. Azar, L: On some extensions of Hardy-Hilbert's inequality and applications. J. Inequal. Appl. 2009, 546829 (2009)

12. Arpad, B, Choonghong, O: Best constant for certain multilinear integral operator. J. Inequal. Appl. 2006, 28582 (2006)

13. Kuang, JC, Debnath, L: On Hilbert's type inequalities on the weighted Orlicz spaces. Pac. J. Appl. Math. 1(1), 95-103 (2007)

14. Zhong, WY: The Hilbert-type integral inequality with a homogeneous kernel of Lambda-degree. J. Inequal. Appl. 2008, 917392 (2008)

15. Hong, Y: On Hardy-Hilbert integral inequalities with some parameters. J. Inequal. Pure Appl. Math. 6(4), 92 (2005)

16. Zhong, WY, Yang, BC: On multiple Hardy-Hilbert's integral inequality with kernel. J. Inequal. Appl. 2007, 27962 (2007). doi:10.1155/2007/27

17. Yang, BC, Krnić, M: On the norm of a multi-dimensional Hilbert-type operator. Sarajevo J. Math. 7(20), $223-243$ (2011)

18. Krnić, M, Pečarić, JE, Vuković, P: On some higher-dimensional Hilbert's and Hardy-Hilbert's type integral inequalities with parameters. Math. Inequal. Appl. 11, 701-716 (2008)

19. Krnić, M, Vuković, P: On a multidimensional version of the Hilbert-type inequality. Anal. Math. 38, 291-303 (2012)

20. Rassias, MT, Yang, BC: On half-discrete Hilbert's inequality. Appl. Math. Comput. 220, 75-93 (2013)

21. Rassias, MT, Yang, BC: A multidimensional half-discrete Hilbert-type inequality and the Riemann zeta function. Appl. Math. Comput. 225, 263-277 (2013)

22. Rassias, MT, Yang, BC: On a multidimensional half-discrete Hilbert-type inequality related to the hyperbolic cotangent function. Appl. Math. Comput. 242, 800-813 (2014)

23. Rassias, MT, Yang, BC: On a multidimensional Hilbert-type integral inequality associated to the gamma function. Appl. Math. Comput. 249, 408-418 (2014)

24. Li, YJ, He, B: On inequalities of Hilbert's type. Bull. Aust. Math. Soc. 76(1), 1-13 (2007)

25. Kuang, JC: Applied Inequalities. Shangdong Science Technic Press, Jinan (2004)

\section{Submit your manuscript to a SpringerOpen ${ }^{\ominus}$ journal and benefit from:}

- Convenient online submission

Rigorous peer review

- Immediate publication on acceptance

- Open access: articles freely available online

- High visibility within the field

- Retaining the copyright to your article 